\title{
Runge-Kutta solutions of stiff differential equations near stationary points
}

\section{Report}

\section{Author(s):}

Lubich, Christian; Nipp, Kaspar; Stoffer, D.

Publication date:

1993-04

\section{Permanent link:}

https://doi.org/10.3929/ethz-a-004283920

\section{Rights / license:}

In Copyright - Non-Commercial Use Permitted

\section{Originally published in:}

SAM Research Report 1993-01 


\title{
Runge-Kutta solutions of stiff differential equations near stationary points
}

\author{
Ch. Lubich ${ }^{1}$, K. Nipp, D. Stoffer
}

Research Report No. 93-01

April 1993

Seminar für Angewandte Mathematik

Eidgenössische Technische Hochschule

CH-8092 Zürich

Switzerland

\footnotetext{
${ }^{1}$ Institut für Angewandte Mathematik und Statistik, Universität Würzburg, Am Hubland, D-W-8700 Würzburg, Germany
} 


\title{
Runge-Kutta solutions of stiff differential equations near stationary points
}

Ch. Lubich ${ }^{1}$, K. Nipp, D. Stoffer

Seminar für Angewandte Mathematik

Eidgenössische Technische Hochschule

CH-8092 Zürich

Switzerland

Research Report No. 93-01

April 1993

\begin{abstract}
Runge-Kutta methods applied to stiff systems in singular perturbation form are shown to give accurate approximations of phase portraits near hyperbolic stationary points. We prove that Runge-Kutta solutions shadow solutions of the differential equation over arbitrarily long time intervals, and vice versa. Sharp error estimates are derived. The proof uses attractive invariant manifolds to reduce the problem to the nonstiff case which was previously studied by Beyn.
\end{abstract}

Keywords: Long-time error bounds, implicit Runge-Kutta method, stiff ODE, singular perturbation problem, hyperbolic equilibrium, shadowing, invariant manifold.

Subject Classification: 65L05

\section{Runge-Kutta solutions of stiff differential equations near stationary points}

\author{
Ch. Lubich, K. Nipp, D. Stoffer
}

\footnotetext{
${ }^{1}$ Institut für Angewandte Mathematik und Statistik, Universität Würzburg, Am Hubland, D-W-8700 Würzburg, Germany
} 
In the literature on the long-time behaviour of numerical solutions of differential equations, there is a remarkable result of Beyn [2] which says that Runge-Kutta methods (and also other one-step methods and multistep methods) give accurate approximations of phase portraits near hyperbolic equilibria, such as saddle points. Stated in more detail: In a sufficiently small neighbourhood of the stationary point, every Runge-Kutta solution obtained with sufficiently small step size approximates some solution of the differential equation, and conversely, every solution in this neighbourhood is approximated by some Runge-Kutta solution, independently of the time interval which may be arbitrarily large. The approximation order is what one would expect for integration over bounded time intervals. The starting values of the solution and of its long-time approximant usually do not coincide. We also refer to the survey articles of Beyn [3] and Sanz-Serna and Larsson [17] which put this and related results into perspective. The result is meaningful in nonstiff situations: With increasing norm of the Jacobian, both the diameter of the neighbourhood and the maximum permitted stepsize shrink to zero in Beyn's result, and the approximation properties deteriorate.

In the present paper we study Runge-Kutta solutions near hyperbolic stationary points of stiff problems in singular perturbation form. We prove a Beyn type result which is valid in a neighbourhood of the equilibrium and for stepsizes that are not restricted by the small stiffness parameter $\epsilon$, and which gives optimal-order error estimates. In Hairer, Lubich, and Roche [7] precise error bounds were obtained for Runge-Kutta approximations of "smooth" solutions of singularly perturbed problems over bounded time intervals. We will show the same approximation order as in [7] for the present problem. In Nipp and Stoffer [15], techniques of [14] and [7] were combined to study the existence and properties of an attractive invariant manifold for Runge-Kutta solutions of singularly perturbed problems. This was used to give an alternative proof of the error bounds of [7]. The invariant manifold results permit us to handle the initial rapid change of general solutions and the associated difficulty that in stiff problems the local error is not small in a whole neighbourhood of the stationary point.

The technique of proof used here aims at reducing the discrete and continuous systems to their invariant manifolds, on which the dynamics are described by nonstiff equations. These techniques permit to infer also other long-time properties of numerical solutions of stiff systems from known results for the nonstiff case. Typical examples are the persistence of hyperbolic periodic orbits and the approximation of attracting sets, which were studied in the nonstiff case by Eirola [5] (among others) and Kloeden and Lorenz [9], respectively. A related idea was recently used by Stoffer [18], where the results of [5] and [9] were extended from one-step methods to general linear methods with the aid of attractive invariant manifolds. 
point of time and space discretizations of semilinear parabolic partial differential equations, which is another "stiff" problem. Larsson and Sanz-Serna [11] obtained sharp estimates for piecewise linear finite element space discretizations. Alouges and Debussche [1] gave a (sub-optimal) extension of Beyn's result for time discretization by the implicit Euler method. Their estimates can be improved to first-order error bounds by combining the techniques of [11] and [10]. However, better than first-order error estimates do not seem to be attainable for higher-order Runge-Kutta methods by using these arguments. One might expect that higher-order estimates require a study of existence and properties of (approximate) inertial manifolds, to be used in a way similar to the attractive invariant manifolds of the present paper.

The paper is organized as follows: In Section 2 we state our main result (Theorem 1) on the behaviour of Runge-Kutta solutions of singularly perturbed problems near a hyperbolic equilibrium point. To prepare for the proof, we review the essentials of Beyn's result for the nonstiff case in Section 3. In Section 4 we discuss some aspects of attractive invariant manifolds for singularly perturbed problems. In Sections 5 and 6 we prove Theorem 1 . To make the proof more transparent, we first derive a slightly weakened bound in Section 5 , and we improve it in Section 6.

\section{Statement of the result}

We consider the singularly perturbed problem

$$
\begin{aligned}
\frac{d y}{d t} & =f(y, z) \\
\epsilon \frac{d z}{d t} & =g(y, z), \quad 0<\epsilon \ll 1,
\end{aligned}
$$

in the neighbourhood of a stationary point, which we may assume to be situated at the origin. Thus $f(0,0)=0$ and $g(0,0)=0$. The functions $f$ and $g$ are assumed to be sufficiently differentiable. As usual in singular perturbation theory, we impose the following condition for Eq.(2.1):

All eigenvalues of $g_{z}(0,0)$ have negative real part.

Further we assume that for small $\epsilon$ the stationary point is hyperbolic, that is, the Jacobian of the system (2.1) at the stationary point has no eigenvalues on the imaginary axis. It is easily verified that this is equivalent to the following condition:

$$
\left(f_{y}-f_{z} g_{z}^{-1} g_{y}\right)(0,0) \text { has no eigenvalues on the imaginary axis. }
$$


size $h \geq \epsilon$ :

$$
y_{n+1}=y_{n}+h \sum_{j=1}^{s} b_{j} Y_{n j}^{\prime}, \quad z_{n+1}=z_{n}+h \sum_{j=1}^{s} b_{j} Z_{n j}^{\prime}
$$

with internal stages $(i=1, \ldots, s)$

$$
Y_{n i}=y_{n}+h \sum_{j=1}^{s} a_{i j} Y_{n j}^{\prime}, \quad Z_{n i}=z_{n}+h \sum_{j=1}^{s} a_{i j} Z_{n j}^{\prime}
$$

satisfying relations of the form of (2.1):

$$
\begin{aligned}
& Y_{n i}^{\prime}=f\left(Y_{n i}, Z_{n i}\right) \\
& \epsilon Z_{n i}^{\prime}=g\left(Y_{n i}, Z_{n i}\right) .
\end{aligned}
$$

We will make the method assumptions of [7]: The Runge-Kutta method is A-stable, i.e., the stability function

$$
R(w)=1+w b^{T}(I-w \mathcal{O})^{-1} 11,
$$

(where $b^{T}=\left(b_{1}, \ldots, b_{s}\right), \mathcal{O}=\left(a_{i j}\right)_{i, j=1}^{s}, 11=(1, \ldots, 1)^{T}$ ) satisfies

$$
|R(w)| \leq 1 \quad \text { for } \operatorname{Re} w \leq 0
$$

We further assume that all eigenvalues of the Runge-Kutta matrix $\mathcal{O}=\left(a_{i j}\right)_{i, j=1}^{s}$ have positive real part, and that $R(\infty)=1-b^{T} \mathcal{O}^{-1} 11$ satisfies

$$
|R(\infty)|<1
$$

If one restricts the attention to $\epsilon \ll h$, then only the invertibility of $\mathcal{O}$ and condition (2.7) are needed. The method has stage order $q$, if

$$
\sum_{j=1}^{s} a_{i j} c_{j}^{k-1}=\frac{c_{i}^{k}}{k} \quad \text { for } k=1, \ldots, q \quad \text { and all } i
$$

Here $c_{i}$ is defined by (2.8) with $k=1$. We assume that the order $p$ of the method when applied to nonstiff ordinary differential equations satisfies $p \geq q+1$. (If $p=q$, this is achieved by reducing $q$ by 1 .)

We will show the following result in this article. 
First assertion: For every Runge-Kutta solution (2.4) with $\left\|\left(y_{n}, z_{n}\right)\right\| \leq r$ for $0 \leq n \leq N$, there exists a solution $(y(t), z(t))$ of (D.1) for $0 \leq t \leq T=N h$, such that for $0 \leq n \leq N$

$$
\begin{aligned}
& \left\|y_{n}-y(n h)\right\| \leq C \cdot\left(h^{p}+\epsilon h^{q+1}+\epsilon \rho^{n}\right) \\
& \left\|z_{n}-z(n h)\right\| \leq \begin{cases}C \cdot\left(h^{p}+\epsilon h^{q}+\rho^{n}\right) & \text { if } a_{s j}=b_{j} \text { for } j=1, \ldots, s, \\
C \cdot\left(h^{q+1}+\rho^{n}\right) & \text { else } .\end{cases}
\end{aligned}
$$

Here $\rho<1$ and $C$ depend only on $f, g, r$ and $h_{0}$, and in particular are independent of $\epsilon$, $h$, and $N$. Moreover, for $\epsilon \ll h$ we have $\rho=|R(\infty)|+O(\epsilon / h)$.

Second assertion: Conversely, for every solution of (D.1) with $\|(y(t), z(t))\| \leq r$ for $0 \leq$ $t \leq T=N h$, there exists a Runge-Kutta solution $\left(y_{n}, z_{n}\right), 0 \leq n \leq N$, satisfying (D.9) with $\rho=e^{-\kappa h / \epsilon}$, with $C$ and $\kappa>0$ independent of $\epsilon, h$, and $N$.

This extends Theorem 3.1 of Beyn [2] to the stiff case. Apart from the rapidly changing initial phase, the approximation order is the same as that established by Hairer, Lubich, and Roche [7] for Runge-Kutta approximations of "smooth" solutions of (2.1) on bounded time intervals. An essential aspect of Theorem 1 is the fact that the estimates remain uniform as the integration interval becomes large. It is again possible to infer the existence of stable and unstable manifolds of the Runge-Kutta scheme which are close to those of the differential equation, similarly as in Beyn [2] for the nonstiff case, cf. also Nipp and Stoffer [14].

\section{A review of the nonstiff case}

Consider the (nonstiff) differential equation

$$
\frac{d x}{d t}=f(x)
$$

with $x=0$ being a hyperbolic equilibrium. Beyn [2], Thm. 3.1, has shown the following for numerical solutions obtained by applying a Runge-Kutta method of order $p$ to Eq. (3.1): For every RK-solution $\left(x_{n}\right), 0 \leq n \leq N$, which stays in a sufficiently small neighbourhood $U$ of the stationary point, there is a solution $x(t)$ of Eq. (3.1) satisfying $\left\|x_{n}-x(n h)\right\| \leq C h^{p}$. Conversely, for every solution $x(t)$ in $U, 0 \leq t \leq T=N h$, there is a RK-solution $\left(x_{n}\right)$ with $\left\|x_{n}-x(n h)\right\| \leq C h^{p}$. The constant $C$ is independent of $h$ and $N$.

This result of Beyn can be viewed as a consequence of the stability lemma stated below. Lemma 2 will also be used in the proof of Theorem 1. 


$$
A=V\left(\begin{array}{ll}
A^{+} & 0 \\
0 & A^{-}
\end{array}\right) V^{-1}
$$

where all eigenvalues of $A^{+}\left(A^{-}\right)$have positive (negative) real part. We use the corresponding spectral projections

$$
P^{+}=V \Pi^{+} V^{-1}, \quad P^{-}=V \Pi^{-} V^{-1}
$$

where

$$
\Pi^{+} v=\left(\begin{array}{c}
v^{+} \\
0
\end{array}\right), \Pi^{-} v=\left(\begin{array}{l}
0 \\
v^{-}
\end{array}\right) \quad \text { for } \quad v=\left(\begin{array}{c}
v^{+} \\
v^{-}
\end{array}\right)
$$

A Runge-Kutta method applied to the differential equation $d x / d t=A x+\varphi(x)$ gives a recursion

$$
x_{n+1}=R(h A) x_{n}+h \phi\left(x_{n}\right),
$$

where $R(w)$ is the stability function $(2.5)$, and $\phi$ is some $h$-dependent function related to $\varphi$, with Lipschitz constant $L_{\phi}=\left(\sum_{i=1}^{s}\left|b_{i}\right|+O(h)\right) L_{\varphi}$. We also consider a perturbed variant

$$
\widetilde{x}_{n+1}=R(h A) \widetilde{x}_{n}+h \phi\left(\widetilde{x}_{n}\right)+h \delta_{n} .
$$

Note that if we take $\tilde{x}_{n}=x(n h)$, where $x(t)$ is a solution of $d x / d t=A x+\varphi(x)$, then $h \delta_{n}$ is the local error, which is $O\left(h^{p+1}\right)$ uniformly in bounded neighbourhoods of the stationary point.

Lemma 2. Assume $\phi(0)=0$ and $\left\|\phi^{\prime}(x)\right\| \leq \ell$ for $\|x\| \leq 2 r$. Let $\left(\tilde{x}_{n}\right)$ with $\left\|\tilde{x}_{n}\right\| \leq r$ for $0 \leq n \leq N$ satisfy (3.5). Let $\delta$ and $\beta$ denote bounds

$$
\begin{gathered}
\left\|\delta_{n}\right\| \leq \delta \quad \text { for } 0 \leq n \leq N \\
\left\|P^{-}\left(x_{0}-\tilde{x}_{0}\right)\right\|+\left\|P^{+}\left(x_{N}-\tilde{x}_{N}\right)\right\| \leq \beta
\end{gathered}
$$

If $\ell, \delta, \beta$, and $h$ are sufficiently small, then equation (3.4) has a unique solution $\left(x_{n}\right)$ with prescribed boundary values $P^{-} x_{0}$ and $P^{+} x_{N}$, which satisfies

$$
\left\|x_{n}-\tilde{x}_{n}\right\| \leq C \cdot(\beta+\delta) \quad \text { for } 0 \leq n \leq N
$$

The constant $C$ is independent of $h$ and $N$.

Proof. Since $R(w)$ is an approximation of the exponential function, we have the bounds

$$
\left\|R\left(h A^{-}\right)^{n}\right\| \leq C \cdot e^{-\alpha h n}, \quad\left\|R\left(h A^{+}\right)^{-n}\right\| \leq C \cdot e^{-\alpha h n}, \quad n \geq 0
$$


$V\left(\begin{array}{c}x_{n}^{+} \\ x_{n}^{-}\end{array}\right)$and $\phi=V\left(\begin{array}{c}\phi^{+} \\ \phi^{-}\end{array}\right)$

$$
\begin{aligned}
& x_{n}^{-}=R\left(h A^{-}\right)^{n} x_{0}^{-}+h \sum_{j=0}^{n-1} R\left(h A^{-}\right)^{n-j-1} \phi^{-}\left(x_{j}\right), \\
& x_{n}^{+}=R\left(h A^{+}\right)^{n-N} x_{N}^{+}-h \sum_{j=n}^{N-1} R\left(h A^{+}\right)^{n-j-1} \phi^{+}\left(x_{j}\right),
\end{aligned}
$$

and similarly for $\widetilde{x}_{n}$. As the Lipschitz constant of $\phi$ can be kept as small as we please, the result follows from the Banach contraction principle (cf. also [11]).

\section{Attractive invariant manifolds}

One difficulty in extending Beyn's result to the stiff problem (2.1), is that the local error is not small for arbitrary starting values near the stationary point. The reason is that general solutions of (2.1) undergo rapid initial changes as is shown in the socalled Tikhonov-Levinson theory of singularly perturbed systems. A description of this theory may be found e.g. in the text-books [19], [16] and also [8]. As is known from [4], [6], [12], [13], there is a smooth attractive invariant manifold $M_{\epsilon}=\left\{(y, z) \mid z=s_{\epsilon}(y)\right\}$ (locally near the stationary point, which itself is on $M_{\epsilon}$ ). Solutions of $(2.1)$ starting on $M_{\epsilon}$ remain on $M_{\epsilon}$ and are "smooth" in the sense that arbitrarily many derivatives are bounded independently of $\epsilon$. An arbitrary solution $(y(t), z(t))$ of $(2.1)$ near $(0,0)$ quickly approaches a solution on $M_{\epsilon}$ : There is a solution $(\tilde{y}(t), \tilde{z}(t))$ on $M_{\epsilon}$ such that

$$
\|y(t)-\widetilde{y}(t)\|+\epsilon \cdot\|z(t)-\widetilde{z}(t)\| \leq C \cdot \epsilon e^{-\kappa t / \epsilon}, \quad 0 \leq t \leq T
$$

with some constants $C$ and $\kappa>0$ which do not depend on $\epsilon$ and $T$ ("property of asymptotic phase"). If $(y(t), z(t))=\left(y(t), s_{\epsilon}(y(t))\right)$ is a solution of Eq. (2.1) on $M_{\epsilon}$ then $y(t)$ is a solution of the (nonstiff) differential equation

$$
\frac{d y}{d t}=f\left(y, s_{\epsilon}(y)\right)
$$

which has $y=0$ as an equilibrium. The function $s_{\epsilon}$ depends smoothly on $\epsilon \geq 0$. For $\epsilon=0$ we denote by $s_{0}(y)=z$ the locally unique solution of the "reduced system" $0=g(y, z)$. In particular, we have $s_{\epsilon}^{\prime}(0)=s_{0}^{\prime}(0)+O(\epsilon)=-\left(g_{z}^{-1} g_{y}\right)(0,0)+O(\epsilon)$. Hence, the Jacobian of Eq. (4.2) at $y=0$ is

$$
\left(f_{y}-f_{z} g_{z}^{-1} g_{y}\right)(0,0)+O(\epsilon) .
$$

By condition (2.3), this matrix has no eigenvalues on the imaginary axis for small $\epsilon$. 
attractive invariant manifold $M_{\epsilon, h}=\left\{(y, z) \mid z=s_{\epsilon, h}(y)\right\}$ for the Runge-Kutta solutions (2.4). $M_{\epsilon, h}$ is close to $M_{\epsilon}$ :

$$
\left\|s_{\epsilon, h}(y)-s_{\epsilon}(y)\right\| \leq \begin{cases}C \epsilon h^{q} & \text { if } b_{i}=a_{s i} \text { for all } i \\ C h^{q+1} & \text { else }\end{cases}
$$

Again, the property of asymptotic phase states that for every $\left(y_{0}, z_{0}\right)$ in an $h$ - and $\epsilon$ independent neighbourhood of $M_{\epsilon, h}$, there exists $\left(\widetilde{y}_{0}, \widetilde{z}_{0}\right) \in M_{\epsilon, h}$ such that the corresponding Runge-Kutta solutions satisfy

$$
\left\|y_{n}-\tilde{y}_{n}\right\|+\epsilon \cdot\left\|z_{n}-\widetilde{z}_{n}\right\| \leq C \cdot \epsilon \rho^{n}, \quad 0 \leq n \leq N
$$

where $\rho<1$ and $C$ do not depend on $\epsilon, h$, and $N$. For $\epsilon \ll h$ we have $\rho=|R(\infty)|+O(\epsilon / h)$.

Remark. The above assertions are a slightly generalized and sharpened version of Theorem 3 of [15]. There, the factor $\epsilon$ in the estimate of the $y$-component in (4.4) is not stated explicitly. The result in [15] is formulated for $\epsilon \ll h$, but it extends to $\epsilon \leq h$ under the stronger method assumptions that we have imposed. This can be shown using the estimates in Section 5 of [7].

\section{Proof of Theorem 1 (slightly weakened estimate)}

The basic idea is to reduce the stiff case to the nonstiff case by means of the invariant manifolds $M_{\epsilon, h}$ and $M_{\epsilon}$. Reducing the discrete and continuous systems to the corresponding invariant manifolds, the dynamics only depend on the nonstiff $y$-equation. More precisely, we proceed as follows to prove the first assertion of Theorem 1: Let $\left(y_{n}, z_{n}\right), 0 \leq n \leq N$, be a given $R K$-solution of Eq.(2.1) staying in an $r$-neighbourhood of $(0,0), r$ sufficiently small. We first take the $R K$-solution $\left(\tilde{y}_{n}, \tilde{z}_{n}\right)$ on $M_{\epsilon, h}$ with the same "asymptotic phase" (see (4.4)). Then, using $M_{\epsilon}$ we construct a $R K$-solution $\left(\eta_{n}\right)$ of the nonstiff system (4.2) "shadowing" $\left(\tilde{y}_{n}\right)$. In a third step, we apply Beyn's result to Eq.(4.2) in order to establish the claimed solution $(y(t), z(t))=\left(y(t), s_{\epsilon}(y(t)), 0 \leq t \leq T=N h\right.$, of Eq.(2.1). Conversely, for the second assertion of Theorem 1: Given a solution $(y(t), z(t))$ of Eq.(2.1), we first take the solution $(\tilde{y}(t), \tilde{z}(t))$ on $M_{\epsilon}$ with the same "asymptotic phase" (see (4.1)). Then, by means of Beyn's result there is a $R K$-solution $\left(\eta_{n}\right)$ of Eq.(4.2) staying close to $(\tilde{y}(n h))$. Finally, we construct a $R K$-solution $\left(y_{n}, z_{n}\right)=\left(y_{n}, s_{\epsilon, h}\left(y_{n}\right)\right)$ of Eq. $(2.1)$ on $M_{\epsilon, h}$ "shadowing" $\left(\eta_{n}, s_{\epsilon}\left(\eta_{n}\right)\right)$.

Proof of the first assertion: We estimate

$$
\left\|y_{n}-y(n h)\right\| \leq\left\|y_{n}-\tilde{y}_{n}\right\|+\left\|\tilde{y}_{n}-\eta_{n}\right\|+\left\|\eta_{n}-y(n h)\right\|
$$

in three steps. 
2) To simplify this step we show first that $\widetilde{y}_{n}-\eta_{n}=O\left(\epsilon h^{q}\right)$. The stronger estimate $O\left(\epsilon h^{q+1}\right)$ will be obtained in the following section.

In order to apply Lemma 2 we need the estimate given in the following lemma.

Lemma 3. Let $y_{0}$ be in an r-neighbourhood of 0 . Apply one step of the RK-method to Eq. (4.D) taking $\eta_{0}=y_{0}$ to $\eta_{1}$. Let $\left(y_{1}, z_{1}\right)$ denote the result of one step of the $R K$-method applied to Eq. (2.1) with starting value $\left(y_{0}, z_{0}\right)=\left(y_{0}, s_{\epsilon, h}\left(y_{0}\right)\right) \in M_{\epsilon, h}$. Then

$$
y_{1}-\eta_{1}=O\left(\epsilon h^{q+1}\right)
$$

holds for $0<\epsilon \leq h \leq h_{0}$, uniformly for $\left\|y_{0}\right\| \leq r$.

Proof. (a) Let $\hat{Y}_{i}(i=1, \ldots, s)$ denote the internal stages of the RK-method applied to Eq. (4.2). Putting

$$
\widehat{Z}_{i}=s_{\epsilon}\left(\hat{Y}_{i}\right)
$$

we then have

$$
\eta_{1}=\eta_{0}+h \sum_{j=1}^{s} b_{j} f\left(\widehat{Y}_{j}, \widehat{Z}_{j}\right), \quad \widehat{Y}_{i}=\eta_{0}+h \sum_{j=1}^{s} a_{i j} f\left(\widehat{Y}_{j}, \widehat{Z}_{j}\right)
$$

We will show in part (b) of the proof that we have, with $\zeta_{0}=s_{\epsilon}\left(\eta_{0}\right)$,

$$
\epsilon\left(\hat{Z}_{i}-\zeta_{0}\right)=h \sum_{j=1}^{s} a_{i j} g\left(\hat{Y}_{j}, \hat{Z}_{j}\right)+O\left(\epsilon h^{q+1}\right) .
$$

On the other hand, the internal stages $Y_{i}, Z_{i}$ of the RK-method applied to Eq. (2.1) satisfy (2.4). The starting values are $\eta_{0}=y_{0}$, and $\zeta_{0}=z_{0}+O\left(h^{q+1}\right)$ by (4.3). We now apply Lemma 6 of [7] (with defects $\delta_{i}=0$ and $h \theta_{i}=O\left(\epsilon h^{q+1}\right)$ by (5.2)). This perturbation lemma gives us the bounds

$$
\hat{Y}_{i}-Y_{i}=O\left(\epsilon h^{q+1}\right), \quad \hat{Z}_{i}-Z_{i}=O\left(\epsilon h^{q}\right)
$$

Using $\eta_{0}=y_{0}$ and the Lipschitz boundedness of $f$ in the equation defining $\eta_{1}$, we thus get the estimate stated in Lemma 3.

(b) The key to proving (5.2) is the identity

$$
\epsilon s_{\epsilon}^{\prime}(y) f\left(y, s_{\epsilon}(y)\right)=g\left(y, s_{\epsilon}(y)\right) \quad \text { for all } y(\text { near } 0)
$$

which is obtained from the second equation in (2.1) restricted to the invariant manifold $M_{\epsilon}: z=s_{\epsilon}(y)$. 
Hence,

$$
\hat{Y}_{i}-y\left(c_{i} h\right)=O\left(h^{q+1}\right), \quad \hat{Z}_{i}-z\left(c_{i} h\right)=O\left(h^{q+1}\right) .
$$

$$
\begin{aligned}
& h \sum_{j=1}^{s} a_{i j} g\left(\hat{Y}_{j}, \hat{Z}_{j}\right)=\epsilon h \sum_{j=1}^{s} a_{i j} s_{\epsilon}^{\prime}\left(\hat{Y}_{j}\right) f\left(\hat{Y}_{j}, s_{\epsilon}\left(\hat{Y}_{j}\right)\right) \\
& =\epsilon\left(\int_{0}^{c_{i} h} s_{\epsilon}^{\prime}(y(t)) f\left(y(t), s_{\epsilon}(y(t))\right) d t+O\left(h^{q+1}\right)\right)=\epsilon\left(\int_{0}^{c_{i} h} \frac{d z}{d t}(t) d t+O\left(h^{q+1}\right)\right) \\
& =\epsilon\left(z\left(c_{i} h\right)-z(0)+O\left(h^{q+1}\right)\right)=\epsilon\left(\hat{Z}_{i}-\zeta_{0}+O\left(h^{q+1}\right)\right),
\end{aligned}
$$

which gives the desired relation (5.2).

Remark. The result of Lemma 3 may also be obtained directly by using the estimate $Z_{i}-s_{\epsilon}\left(Y_{i}\right)=O\left(\epsilon h^{q}\right)$ given in Remark 6 of Nipp and Stoffer [15]. On the other hand, this estimate is also a consequence of the estimates derived in the above proof.

As shown in Section 4, Eq.(4.2) may be written as

$$
\frac{d y}{d t}=A y+\varphi(y)
$$

where $A=\left(f_{y}-f_{z} g_{z}^{-1} g_{y}\right)(0,0)$ is hyperbolic and $\varphi$ has Lipschitz constant $O(r)+O(\epsilon)=$ $O(r)$ (assuming $\epsilon<r$ ). Hence, applying the $R K$-method to Eq.(4.2) yields a recursion of the form

$$
\eta_{n+1}=R(h A) \eta_{n}+h \phi\left(\eta_{n}\right)
$$

where the Lipschitz constant of $\phi$ is again $O(r)$. Lemma 3 shows that the RK-solution $\left(\widetilde{y}_{n}, \widetilde{z}_{n}\right)=\left(\tilde{y}_{n}, s_{\epsilon, h}\left(\tilde{y}_{n}\right)\right) \in M_{\epsilon, h}$ of the singularly perturbed system (2.1) satisfies

$$
\tilde{y}_{n+1}=R(h A) \tilde{y}_{n}+h \phi\left(\tilde{y}_{n}\right)+O\left(\epsilon h^{q+1}\right) .
$$

We may thus apply Lemma 2 with $\eta_{n}$ in the role of $x_{n}$, with $\tilde{y}_{n}$ in the role of $\tilde{x}_{n}$ and with $h \delta_{n}=O\left(\epsilon h^{q+1}\right)$. With boundary values chosen as

$$
P^{-} \eta_{0}=P^{-} \tilde{y}_{0}, \quad P^{+} \eta_{N}=P^{+} \tilde{y}_{N},
$$

Lemma 2 gives us a $R K$-solution $\left(\eta_{n}\right)$ of Eq.(4.2) which satisfies

$$
\left\|\tilde{y}_{n}-\eta_{n}\right\| \leq C \epsilon h^{q} \quad \text { for } 0 \leq n \leq N
$$

3) Beyn's result applied to Eq. (4.2) now yields: There is a solution $y(t)$ satisfying

$$
\left\|\eta_{n}-y(n h)\right\| \leq C h^{p} \quad \text { for } 0 \leq n \leq N .
$$

The constant $C$ is again independent of $\epsilon$, because the right-hand side of Eq. (4.2) depends smoothly on $\epsilon \geq 0$. 


$$
\left\|y_{n}-y(n h)\right\| \leq C\left(\epsilon \rho^{n}+\epsilon h^{q}+h^{p}\right)
$$

It remains to estimate the $z$-components. We have

$$
\left\|z_{n}-z(n h)\right\| \leq\left\|z_{n}-\tilde{z}_{n}\right\|+\left\|\tilde{z}_{n}-z(n h)\right\|, \quad 0 \leq n \leq N
$$

where $\left(\tilde{y}_{n}, \tilde{z}_{n}\right) \in M_{\epsilon, h},(y(t), z(t)) \in M_{\epsilon}$. We thus get

$$
\left\|z_{n}-z(n h)\right\| \leq\left\|z_{n}-\tilde{z}_{n}\right\|+\left\|s_{\epsilon, h}\left(\tilde{y}_{n}\right)-s_{\epsilon, h}(y(n h))\right\|+\left\|s_{\epsilon, h}(y(n h))-s_{\epsilon}(y(n h))\right\| .
$$

The property of asymptotic phase (4.4) yields $\left\|z_{n}-\widetilde{z}_{n}\right\| \leq C \rho^{n}$; steps 2), 3) imply $\left\|s_{\epsilon, h}\left(\tilde{y}_{n}\right)-s_{\epsilon, h}(y(n h))\right\| \leq C\left(\epsilon h^{q}+h^{p}\right)$, and the last term is bounded by (4.3). Combining these estimates we obtain

$$
\left\|z_{n}-z(n h)\right\| \leq \begin{cases}C\left(\rho^{n}+\epsilon h^{q}+h^{p}\right) & \text { if } b_{i}=a_{s i}, \\ C\left(\rho^{n}+h^{q+1}\right) & \text { else. }\end{cases}
$$

This completes the proof of the first assertion of Theorem 1, except that the $O\left(\epsilon h^{q}\right)$ term in the error estimate of the $y$-component is not yet optimal. This term will be improved to $O\left(\epsilon h^{q+1}\right)$ in the following section.

Proof of the second assertion: The three steps to construct a $R K$-solution $\left(y_{n}, z_{n}\right)$ on $M_{\epsilon, h}$ are sketched in the beginning of Section 5. We do not give the details, since they are almost the same as in the proof of the first assertion.

\section{Proof of Theorem 1 (refined estimate)}

To complete the proof of Theorem 1, we show that the difference between the sequences $\left(\tilde{y}_{n}\right)$ and $\left(\eta_{n}\right)$ constructed in Section 5 is $O\left(\epsilon h^{q+1}\right)$. From (5.4) we know already that the difference is $O\left(\epsilon h^{q}\right)$. To improve this estimate, we need a refined version of Lemma 3 . In the situation of Lemma 3 , we would of course have $y_{1}=\eta_{1}$ if the function $f$ were independent of $z$, i.e., if $f_{z} \equiv 0$. For $f_{z}\left(y_{0}, s_{\epsilon}\left(y_{0}\right)\right)=O(h)$, tracing the proof of Lemma 3 shows that we still have $y_{1}-\eta_{1}=O\left(\epsilon h^{q+2}\right)$ which improves the estimate of Lemma 3 by the missing factor $h$. The idea for improving the estimate in the general case now consists in transforming variables

$$
u=y-\epsilon B z, \quad v=z
$$

where the constant matrix $B$ is chosen such that Eq. (2.1) written in the variables $(u, v)$,

$$
\begin{aligned}
\frac{d u}{d t} & =f(u+\epsilon B v, v)-B g(u+\epsilon B v, v) & =: F(u, v) \\
\epsilon \frac{d v}{d t} & =g(u+\epsilon B v, v) & =: G(u, v)
\end{aligned}
$$


Lemma 4. Let the situation of Lemma 3 be given, except that the initial condition $y_{0}=\eta_{0}$ is replaced by

$$
y_{0}-\epsilon B s_{\epsilon, h}\left(y_{0}\right)=\eta_{0}-\epsilon B s_{\epsilon}\left(\eta_{0}\right),
$$

with $B=\left(f_{z} g_{z}^{-1}\right)\left(y_{0}, z_{0}\right)+O(h)$. Then,

$$
y_{1}-\epsilon B s_{\epsilon, h}\left(y_{1}\right)=\eta_{1}-\epsilon B s_{\epsilon}\left(\eta_{1}\right)+O\left(\epsilon h^{q+2}\right)+O\left(h^{p+1}\right) .
$$

Proof. Since the RK-method is invariant under linear transformations of variables, we have that $\left(u_{1}, v_{1}\right):=\left(y_{1}-\epsilon B z_{1}, z_{1}\right)$ is the result of one step of the RK-method applied to Eq. (6.1) with starting value $\left(u_{0}, v_{0}\right):=\left(y_{0}-\epsilon B z_{0}, z_{0}\right)$.

The invariant manifold of the singularly perturbed system $(6.1)$ is parametrized by $v=$ $S_{\epsilon}(u)$ with $S_{\epsilon}$ defined by $S_{\epsilon}(u)=s_{\epsilon}(y)$ for $u=y-\epsilon B s_{\epsilon}(y)$. Let $\mu_{1}$ denote the result of the RK-method applied to the differential equation (which is the $u$-version of Eq. (4.2))

$$
\frac{d u}{d t}=F\left(u, S_{\epsilon}(u)\right)
$$

with starting value $\mu_{0}=\eta_{0}-\epsilon B s_{\epsilon}\left(\eta_{0}\right)$. By our assumptions, we have $u_{0}=\mu_{0}$ and $F_{v}\left(u_{0}, v_{0}\right)=O(h)$. By the arguments in the beginning of this section we thus have

$$
u_{1}-\mu_{1}=O\left(\epsilon h^{q+2}\right)
$$

Both $\mu_{1}$ and $\eta_{1}-\epsilon B s_{\epsilon}\left(\eta_{1}\right)$ approximate the solution $u(t)=y(t)-\epsilon B s_{\epsilon}(y(t))$ of Eq. (6.2) with initial value $u(0)=\mu_{0}$ at $t=h$ with an error of size $O\left(h^{p+1}\right)$. Hence we have

$$
\mu_{1}=\eta_{1}-\epsilon B s_{\epsilon}\left(\eta_{1}\right)+O\left(h^{p+1}\right) .
$$

Since $u_{1}=y_{1}-\epsilon B s_{\epsilon, h}\left(y_{1}\right)$, the result follows.

Remark. A more detailed study (using trees and elementary differentials) reveals that the difference between $\mu_{1}$ and $\eta_{1}-\epsilon B s_{\epsilon}\left(\eta_{1}\right)$ is actually $O\left(\epsilon h^{p+1}\right)$. Hence the $O\left(h^{p+1}\right)$ term in Lemma 4 can be omitted. We have not carried this out, because Lemma 4 as stated above is sufficient for our needs.

Let $\left(\tilde{y}_{n}\right)$ and $\left(\eta_{n}\right)$ be the sequences constructed in Section 5. We put

$$
B_{n}=\left(f_{z} g_{z}^{-1}\right)\left(\eta_{n}, s_{\epsilon}\left(\eta_{n}\right)\right)
$$

and introduce

$$
\tilde{u}_{n}=\tilde{y}_{n}-\epsilon B_{n} s_{\epsilon, h}\left(\tilde{y}_{n}\right), \quad \mu_{n}=\eta_{n}-\epsilon B_{n} s_{\epsilon}\left(\eta_{n}\right)
$$




$$
s_{\epsilon, h}\left(\widetilde{y}_{n}\right)-s_{\epsilon}\left(\eta_{n}\right)=O\left(h^{q+1}\right) .
$$

Inserting the recursion (5.3) for $\eta_{n}$ into (6.3), we get a similar recursion for $\mu_{n}$ :

$$
\mu_{n+1}=R(h A) \mu_{n}+h \phi_{n}\left(\mu_{n}\right)+c_{n}
$$

where $\phi_{n}\left(\mu_{n}\right)=\phi\left(\eta_{n}\right)+h^{-1}\left\{R(h A) \cdot \epsilon B_{n} s_{\epsilon}\left(\eta_{n}\right)-\epsilon B_{n} s_{\epsilon}\left(\eta_{n+1}\right)\right\}$ (with $\eta_{n}$ defined as a function of $\mu_{n}$ via (6.3) and $\eta_{n+1}$ considered as a function of $\eta_{n}$ ) has Lipschitz constant of size $O(r)+O(\epsilon)$ uniformly for all $n$, and $c_{n}=-\epsilon\left(B_{n+1}-B_{n}\right) s_{\epsilon}\left(\eta_{n+1}\right)$. Lemma 4 now gives

$$
\tilde{y}_{n+1}-\epsilon B_{n} s_{\epsilon, h}\left(\tilde{y}_{n+1}\right)=R(h A) \tilde{u}_{n}+h \phi_{n}\left(\tilde{u}_{n}\right)+O\left(\epsilon h^{q+2}\right)+O\left(h^{p+1}\right) .
$$

Together with the estimate $\epsilon\left(B_{n+1}-B_{n}\right)\left(s_{\epsilon, h}\left(\tilde{y}_{n+1}\right)-s_{\epsilon}\left(\eta_{n+1}\right)\right)=O\left(\epsilon h^{q+2}\right)$, see $(6.4)$, this yields

$$
\tilde{u}_{n+1}=R(h A) \tilde{u}_{n}+h \phi_{n}\left(\tilde{u}_{n}\right)+c_{n}+O\left(\epsilon h^{q+2}\right)+O\left(h^{p+1}\right) .
$$

Moreover, the boundary values satisfy

$$
P^{-} \tilde{u}_{0}-P^{-} \mu_{0}=-P^{-} \epsilon B_{0}\left(s_{\epsilon, h}\left(\tilde{y}_{0}\right)-s_{\epsilon}\left(\eta_{0}\right)\right)=O\left(\epsilon h^{q+1}\right)
$$

where we used again (6.4), and similarly also

$$
P^{+} \tilde{u}_{N}-P^{+} \mu_{N}=O\left(\epsilon h^{q+1}\right) .
$$

Lemma 2 (or rather a trivial generalization which allows the nonlinearity to depend on n) now implies

$$
\tilde{u}_{n}-\mu_{n}=O\left(\epsilon h^{q+1}\right)+O\left(h^{p}\right), \quad 0 \leq n \leq N .
$$

Together with (6.3) and (6.4) this gives

$$
\tilde{y}_{n}-\eta_{n}=O\left(\epsilon h^{q+1}\right)+O\left(h^{p}\right), \quad 0 \leq n \leq N .
$$

Using this improved estimate in (5.1) completes the proof of Theorem 1.

\section{References}

[1] F. Alouges, A. Debussche, On the qualitative behavior of the orbits of a parabolic partial differential equation and its discretization in the neighborhood of a hyperbolic fixed point. Numer. Funct. Anal. Optimiz. 12 (1991), 253-269.

[2] W.J. Beyn, On the numerical approximation of phase portraits near stationary points. SIAM J. Numer. Anal. 24 (1987), 1095-1113.

[3] W.J. Beyn, Numerical methods for dynamical systems. In: W. Light (ed.), Advances in Numerical Analysis, Vol. I, Nonlinear partial differential equations and dynamical systems. Clarendon Press, Oxford, 1991, 175-236. 
[5] T. Eirola, Invariant curves of one-step methods. BIT 28 (1988), 113-122.

[6] N. Fenichel, Geometric singular perturbation theory for ordinary differential equations. J. Diff. Eq. 31 (1979), 53-98.

[7] E. Hairer, Ch. Lubich, M. Roche, Error of Runge-Kutta methods for stiff problems studied via differential-algebraic equations. BIT 28 (1988), 678-700.

[8] E. Hairer, G. Wanner, Solving Ordinary Differential Equations II. Stiff and Differential-Algebraic Problems. Springer-Verlag, 1991.

[9] P.E. Kloeden, J. Lorenz, Stable attracting sets in dynamical systems and in their one-step discretizations. SIAM J. Numer. Anal. 23 (1986), 986-995.

[10] S. Larsson, Nonsmooth data error estimates with applications to the long-time behavior of finite element solutions of semilinear parabolic problems. Report 1992-36, Dept. of Math., Chalmers Univ., Göteborg, 1992.

[11] S. Larsson, J.M. Sanz-Serna, The behavior of finite element solutions of semilinear parabolic problems near stationary points. SIAM J. Numer. Anal., to appear.

[12] K. Nipp, Invariant manifolds of singularly perturbed ordinary differential equations. ZAMP 36 (1985), 309-320.

[13] K. Nipp, Smooth attractive invariant manifolds of singularly perturbed ODE's. Report 92-13, SAM, ETH Zürich, 1992.

[14] K. Nipp, D. Stoffer, Attractive invariant manifolds for maps: Existence, smoothness and continuous dependence on the map. Report 92-11, SAM, ETH Zürich, 1992.

[15] K. Nipp, D. Stoffer, Invariant manifolds of numerical integration schemes applied to stiff systems of singular perturbation type - Part I: RK-methods. Report 92-14, SAM, ETH Zürich, 1992.

[16] R.E. O’Malley, Jr., Singular Perturbation Methods for Ordinary Differential Equations. Springer-Verlag, 1991.

[17] J.M. Sanz-Serna, S. Larsson, Shadows, chaos and saddles. Appl. Numer. Math., to appear.

[18] D. Stoffer, General linear methods: connection to one-step methods and invariant curves. Numer. Math. 64 (1993), 395-408.

[19] A.N. Tikhonov, A.B. Vasil'eva, A.G. Sveshnikov, Differential Equations. SpringerVerlag, 1985. 


\begin{tabular}{|c|c|c|}
\hline $93-01$ & $\begin{array}{l}\text { Ch. Lubich, K. Nipp, } \\
\text { D. Stoffer }\end{array}$ & $\begin{array}{l}\text { Runge-Kutta solutions of stiff differential } \\
\text { equations near stationary points }\end{array}$ \\
\hline $92-15$ & N. Botta & $\begin{array}{l}\text { Is the transonic flow around a cylinder always } \\
\text { periodic? }\end{array}$ \\
\hline $92-14$ & K. Nipp, D. Stoffer & $\begin{array}{l}\text { Invariant manifolds of numerical integration } \\
\text { schemes applied to stiff systems of singular } \\
\text { perturbation type - Part I: } R K \text {-methods }\end{array}$ \\
\hline $92-13$ & K. Nipp & $\begin{array}{l}\text { Smooth attractive invariant manifolds of sin- } \\
\text { gularly perturbed ODE's }\end{array}$ \\
\hline $92-12$ & D. Мao & $\begin{array}{l}\text { A Shock Tracking Technique Based on Con- } \\
\text { servation in One Space Dimension }\end{array}$ \\
\hline $92-11$ & K. Nipp, D. Stoffer & $\begin{array}{l}\text { Attractive invariant manifolds for maps: } \\
\text { Existence, smoothness and continuous depen- } \\
\text { dence on the map }\end{array}$ \\
\hline $92-10$ & M. Fey, R. Jeltsch & A Simple Multidimensional Euler Scheme \\
\hline $92-09$ & M. Fey, R. Jeltsch & A New Multidimensional Euler Scheme \\
\hline $92-08$ & $\begin{array}{l}\text { M. Fey, R. Jeltsch, } \\
\text { P. Karmann }\end{array}$ & Numerical solution of a nozzle flow \\
\hline $92-07$ & $\begin{array}{l}\text { M. Fey, R. Jeltsch, } \\
\text { P. Karmann }\end{array}$ & $\begin{array}{l}\text { Special aspects of reacting inviscid blunt } \\
\text { body flow }\end{array}$ \\
\hline 92-06 & $\begin{array}{l}\text { M. Fey, R. Jeltsch, } \\
\text { S. Müller }\end{array}$ & $\begin{array}{l}\text { The influence of a source term, an example: } \\
\text { chemically reacting hypersonic flow }\end{array}$ \\
\hline $92-05$ & N. Botta, J. Sesterhenn & $\begin{array}{l}\text { Deficiencies in the numerical computation of } \\
\text { nozzle flow }\end{array}$ \\
\hline 92-04 & Ch. Lubich & $\begin{array}{l}\text { Integration of stiff mechanical systems by } \\
\text { Runge-Kutta methods }\end{array}$ \\
\hline $92-03$ & M. Fey, R. Jeltsch, S. Müller & Stagnation point analysis \\
\hline $92-02$ & $\begin{array}{l}\text { C. W. Schulz-Rinne, } \\
\text { J. P. Collins, H. M. Glaz }\end{array}$ & $\begin{array}{l}\text { Numerical Solution of the Riemann Problem } \\
\text { for Two-Dimensional Gas Dynamics }\end{array}$ \\
\hline $92-01$ & $\begin{array}{l}\text { R. J. LeVeque, } \\
\text { K. M. Shyue }\end{array}$ & $\begin{array}{l}\text { Shock Tracking Based on High Resolution } \\
\text { Wave Propagation Methods }\end{array}$ \\
\hline $91-10$ & M. Fey, R. Jeltsch & $\begin{array}{l}\text { Influence of numerical diffusion in high tem- } \\
\text { perature flow }\end{array}$ \\
\hline 91-09 & R. J. LeVeque, R. Walder & $\begin{array}{l}\text { Grid Alignment Effects and Rotated Methods } \\
\text { for Computing Complex Flows in Astro- } \\
\text { physics }\end{array}$ \\
\hline $91-08$ & Ch. Lubich, R. Schneider & $\begin{array}{l}\text { Time discretization of parabolic boundary in- } \\
\text { tegral equations }\end{array}$ \\
\hline $91-07$ & M. Pirovino & $\begin{array}{l}\text { On the Definition of Nonlinear Stability for } \\
\text { Numerical Methods }\end{array}$ \\
\hline $91-06$ & $\begin{array}{l}\text { Ch. Lubich, } \\
\text { A. Ostermann }\end{array}$ & $\begin{array}{l}\text { Runge-Kutta Methods for Parabolic Equa- } \\
\text { tions and Convolution Quadrature }\end{array}$ \\
\hline
\end{tabular}

